\title{
Construction of a Mobile Learning Environment Based on Complex Network
}

\author{
https://doi.org/10.3991/ijet.v17i04.29587 \\ Jie Zhao \\ Department of Foreign Studies, North China Electric Power University (Baoding), Baoding, \\ China \\ 52651393@ncepu.edu.cn
}

\begin{abstract}
Fragmentation Learning based on intelligent mobile terminals can generate a large amount of mobile learning trajectory data, and the network formed by these mobile terminals is a very complex network. This paper researched the construction of a mobile learning environment based on complex network, in the hopes of providing a new idea for solving problems in the learning information dissemination of mobile terminals and the integration of mobile learning resources. In the beginning, this paper introduced the concept and features of complex network for mobile learning in the context of an English fragmentation learning environment, built a learning information dissemination model, and proposed an immune strategy under the conditions that terminal nodes won't perform similar repeated learning after completing mobile learning. Then, the paper elaborated on the construction process of a mobile learning environment based on keyword co-occurrence, and established the keyword cooccurrence adjacency matrix and weighted matrix. In the end, experimental results proved the accuracy and usability of the proposed strategy.
\end{abstract}

Keywords-mobile terminal, complex network, mobile learning, learning environment construction

\section{Introduction}

A growing number of learners have now chosen to use intelligent mobile terminals to conduct fragmentation learning as supporting technologies such as Internet, cloud computing, Internet of Things and intelligent terminal devices matured in recent years, such trend has generated massive mobile learning trajectory data covering aspects such as history learning behaviors and interactive learning activities [1-13]. These mobile terminal devices can be connected based on a same learning objective and form a network, which has many complex network features [14-21]. By carefully analyzing these features and making good use of the said network, we can find new ways for solving problems lying in the learning information dissemination of mobile terminals and the integration of mobile learning resources.

Social networks and mobile devices are major tools for reflecting the latest trends in higher education. Simonova and Poulova [22] collected relevant and appropriate data 
for statistics on the types and access frequencies of 19 social networks and the ownership and purposes of use of 12 kinds of mobile devices; the results showed that students have been fully equipped with mobile devices and use them for private purposes, and a high learning efficiency has been achieved. Amaratunga and Ranasinghe [23] created a mechanism to exploit the inherent but haven't been fully utilized functions of mobile devices with a content format specifically designed to maximize opportunistic educational content delivery and take it as a means of disseminating basic educational content. Since wireless network has become the primary infrastructure of digital campus, Chinese colleges and universities are expanding the coverage of campus information network. Chen and Zhu [24] introduced an intelligent algorithm to wireless network communication to optimize and construct an autonomous mobile learning platform for cloud education, they proposed methods for reducing energy loss in wireless communication and finding out the intelligent algorithm most suitable for realizing cloud education via mobile learning platforms. Aiming at the defects that English mobile learning platforms are easily affected by external interference signals and their ability of remote English information transmission is often poor, Zhen [25] put forward a design scheme for English mobile learning platforms based on the GSM-R (Global System for Mobile Communications-Railway) wireless network communication system; they devised the overall framework of an English mobile learning platform, and experimental results suggested that the designed English mobile learning platform can meet students' learning demands, after using it, the students' comprehensive ability has been improved indeed. Zhang and Zhong [26] researched the establishing of learning models via collaborative network inquiry and explored its application in teaching practice, and they attempted to build a mobile learning model of collaborative network inquiry based on big data.

Existing studies concerning mobile learning environment mostly concentrated on the time series of mobile learning trajectory data, none of them talks about directly building complex network for these mobile learning trajectory data. In view of this, based on moving trajectories, this paper presented a secure information dissemination strategy for mobile learning in complex network, and built a mobile learning environment based on keyword co-occurrence. The second chapter of the paper introduced the concept and features of complex network for mobile learning in the context of an English fragmentation learning environment, constructed a learning information dissemination model, and proposed an immune strategy under the conditions that terminal nodes won't perform similar repeated learning after completing mobile learning. The third chapter is the core content of this paper, it elaborated on the construction process of a mobile learning environment based on keyword co-occurrence, and built the keyword co-occurrence adjacency matrix and weighted matrix. In later parts, this paper verified the proposed strategy combining with experimental results, and proved its accuracy and usability. 


\section{Application of complex network in mobile learning environment}

Fragmentation learning on intelligent mobile terminals is the most extensively used learning method for English learning, and all discussions in this paper were based on English learning application scenarios.

A specific mobile learning network can be abstracted as a graph $H=\langle U, R\rangle$ consisting of a point set $U$ and an edge set $R$. Assuming $Y=|U|$ represents the number of terminal nodes and $X=|R|$ represents the number of edges, then for every edge in edge set $R$, there is a pair of nodes in $U$ that correspond to it.

Degree is one of the simple parameters used to describe the topology of mobile learning network. Assuming: $l_{i}$ represents the degree of terminal node $i$ in the network, namely the number of other nodes connected to this terminal node. In a mobile learning network without self-loops and multiple edges, the degree of node $i$ is the total number of edges connected to node $i$; assuming $\langle l\rangle$ represents the average degree of network terminal nodes, namely the mean of degree $l_{i}$ of all terminal nodes $i$ in the network, its calculation formula is:

$$
\langle l\rangle=\frac{\sum_{i \in U} l_{i}}{Y}
$$

In a directed mobile learning network, the degree of each terminal node includes the out-degree and the in-degree, which are represented by $l_{\text {out }}$ and $l_{\text {in }}$, respectively. $l_{\text {out }}$ is equal to the number of edges pointing from a terminal node to other nodes; and $l_{\text {in }}$ is equal to the number of edges pointing from other terminal nodes to this node. Intuitively seen, a node with a larger degree is more important to the entire network than a node with a smaller degree.

The average path length of the mobile learning network has an important impact on network delay, and it is also an important measure and parameter of the network. The average path length is the number of edges on the shortest path between any two terminal nodes $i$ and $j$ in the network, denoted as $\varepsilon_{i j}$; and the average path length of the entire mobile learning network, which is denoted as $\psi$, is the expected value of the sum of all $\varepsilon_{i j}$ values; assuming $Y$ represents the size of the network, then $\psi$ can be calculated by Formula 2:

$$
\psi=\frac{\sum_{i \geq j} \varepsilon_{i j}}{\frac{1}{2} Y(Y+1)}
$$

The clustering coefficient is used to describe the connecting and clustering status of terminal nodes in the network. A larger clustering coefficient indicates tighter local connections of terminal nodes in the network. Assuming: in the network, a terminal node $i$ is connected to other terminal nodes through $l_{i}$ edges, these $l_{i}$ terminal nodes can be defined as the neighbors of terminal node $i$. Then for these neighbors of terminal node $i$, there're at most $l_{i}\left(l_{i}-1\right) / 2$ possible edges that connect these $l_{i}$ terminal nodes with 
each other; assuming $R_{i}$ represents the number of edges that actually exist between these $l_{i}$ terminal nodes, defining the clustering coefficient $\eta_{i}$ of terminal node $i$ as the ratio of $R_{i}$ to the possible number of edges $l_{i}\left(l_{i}-1\right) / 2$, then its value can be calculated by Formula 3:

$$
\eta_{i}=\frac{2 R_{i}}{l_{i}\left(l_{i}-1\right)}
$$

This paper built a learning information dissemination model for mobile learning network based on the epidemic disease spread model. Formula 4 gives the basic differential equations of the epidemic disease spread model:

$$
\left\{\begin{array}{l}
\frac{d e(\tau)}{d \tau}=-u o(t) e(\tau) \\
\frac{d o(\tau)}{d \tau}=u o(\tau) e(\tau)
\end{array}\right.
$$

Assuming: $Y$ is the total size of the mobile learning network; the infected individuals and susceptible individuals in the epidemic disease spread model respectively represent the terminal nodes that have conducted mobile learning and the terminal nodes that need to conduct mobile learning in the mobile learning network. Defining: at time moment $\tau$, the number of infected individuals in the network is represented by $e(\tau)$, and the number of susceptible individuals in the network is represented by $o(\tau)$, then the proportion of individuals in the network that are in the susceptible state $E$ is $e(\tau)=e(\tau) / M$, the proportion of individuals that are in the infected state $O$ is $i(\tau)=i(\tau) / M$, and there is $e(\tau)+i(\tau)=1$. According to actual situations, the dissemination model can reflect the initial characteristics of learning information dissemination in the mobile learning network.

Because for each terminal node in the mobile learning network, after it completes mobile learning for one time, it has the needs to proceed to the mobile learning of next level, that is, all terminal nodes can convert between susceptible state $E$ and infected state $O$. The differential equations of the model can be updated as follows:

$$
\left\{\begin{array}{l}
\frac{d e(\tau)}{d \tau}=-u o(\tau) e(\tau)+\xi o(\tau) \\
\frac{d o(\tau)}{d \tau}=u o(\tau) e(\tau)-\xi o(\tau)
\end{array}\right.
$$

The two models of Formulas 4 and 5 have a common disadvantage, they only consider whether a terminal node is conducting mobile learning or whether mobile learning is required, that is, whether an individual is healthy or infected in the epidemic disease spread model, they haven't considered the situation whether the terminal nodes will conduct similar repeated learning after completing the mobile learning, namely the situation that individuals have acquired immunity in the epidemic disease spread model, which is inconsistent with the actual situation of virus transmission. For this reason, in 
this paper, differential equations of the constructed model were further updated. Assuming: $u$ represents the probability of susceptible nodes being infected due to the information transmission between the susceptible nodes and infected nodes; $\xi$ represents the probability of infected nodes obtaining permanent immunity due to receiving treatment, then there are:

$$
\left\{\begin{array}{l}
\frac{d e(\tau)}{d \tau}=-u o(\tau) e(\tau) \\
\frac{d o(\tau)}{d \tau}=u o(\tau) e(\tau)-\xi o(\tau) \\
\frac{d s(\tau)}{d \tau}=\xi o(\tau)
\end{array}\right.
$$

In the traditional epidemic disease spread model, the individuals' immunity can be obtained by randomly selecting some nodes in the network for immunity, and there is no priority in it. Assuming $\rho$ represents the density of immune terminal nodes, then Formula 7 gives the calculation formula of the critical value of immunity $\rho_{I M}$ :

$$
\rho_{I M}=1-\frac{\mu_{I M}}{\mu}
$$

Formula 8 gives the formula for calculating the critical value of immunity $\rho_{I M}$ of the scale-free network:

$$
\rho_{I M}=1-\frac{\langle l\rangle}{\mu\left\langle l^{2}\right\rangle}
$$

According to above formula, as the average degree of nodes $\left\langle l^{2}\right\rangle$ of the scale-free network tends to infinity, $\rho_{I M}$ tends to 1 . Adopting the above random immunity strategy requires that most terminal nodes in the network to be immunized, the immunity efficiency is relatively low. In this paper, according to the degree of terminal nodes, from large to small, the terminal nodes that need to be immunized were selected, and Formula 9 gives the formula for calculating the critical value of immunity of the corresponding target:

$$
\rho_{I M} \sim r^{-\frac{2}{n \mu}}
$$

The above formula shows that, the immune strategy makes use of the uneven distribution characteristic of scale-free network, the more uneven the mobile learning network is, the more obvious the effect that the terminal nodes won't conduct similar repeated learning after completing the mobile learning. 


\section{Construction of mobile learning environment based on keyword co-occurrence}

This chapter figured out the relationship between research hotspots of English fragmentation learning and hot learning words via constructing a mobile learning environment based on keyword co-occurrence. Figure 1 shows the keyword screening process of English fragmentation learning. After the keyword co-occurrence dataset and highfrequency vocabulary data were determined, the keyword co-occurrence adjacency matrix and weighted matrix were constructed to finally realize the construction of the keyword co-occurrence mobile learning environment.

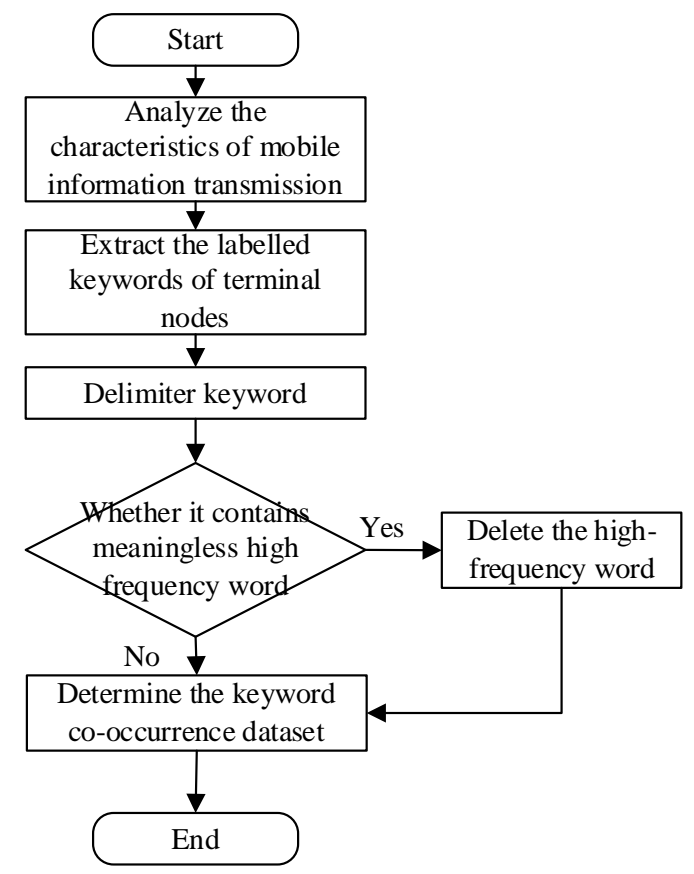

Fig. 1. Keyword screening process of English fragmentation learning

In graph $H<U, R>$, if there is information transmission between two terminal nodes, the co-occurrence frequency is not zero. The $m^{*} m$ matrix $R H$ used to describe the connections between all edges in $R$ can be expressed as Formula 10:

$$
R H=\left[\begin{array}{cccc}
r_{11} & r_{12} & \cdots & r_{m 1} \\
r_{21} & r_{22} & \cdots & r_{m 2} \\
\vdots & \vdots & \ddots & \vdots \\
r_{m 1} & r_{m 2} & \cdots & r_{m m}
\end{array}\right], 1 \leq i, j \leq m
$$


Assuming: $r_{i j}$ represents the edge between terminal nodes $U_{i}$ and $U_{j}$ of labelled keywords. If there is an edge between $U_{i}$ and $U_{j}$, then $r_{i j}$ is 1 ; otherwise, $r_{i j}$ is 0 .

In order to determine the co-occurrence intensity of the labelled keywords of two terminal nodes, it is necessary to simplify the keyword co-occurrence adjacency matrix $R H$ of the original network, only retain the closest connection between terminal nodes, and then construct the final keyword co-occurrence mobile learning environment on this basis. Same as $R H$, the weighted matrix $\omega$ defines a weight $\omega_{i j}$ that is related to edge $r_{i j}$, then there is:

$$
\omega=\left[\begin{array}{cccc}
\omega_{11} & \omega_{12} & \cdots & \omega_{n 1} \\
\omega_{21} & \omega_{22} & \cdots & \omega_{m 2} \\
\vdots & \vdots & \ddots & \vdots \\
\omega_{m 1} & \omega_{m 2} & \cdots & \omega_{m m}
\end{array}\right], 1 \leq i, j \leq m
$$

The $\omega_{i j}$ values on the diagonal are all 0 . If $r_{i j}=0$, then $\omega_{i j}=0$. In this paper, the included angle cosine algorithm was adopted to calculate the weight between the labelled keywords of two terminal nodes. Assuming: $D E_{i j}$ represents the co-occurrence times of keyword $i$ and terminal node $j, F R_{i}$ represents the frequency of occurrence of keyword $i, F R_{i}$ represents the frequency of occurrence of keyword $j$, then there is:

$$
\Gamma\left(D E_{i j}, F R_{i}, F R_{j}\right)=\frac{D E_{i j}}{\sqrt{F R_{i} F R_{j}}}
$$

The size of $\Gamma$ describes the connection intensity between terminal nodes in the keyword co-occurrence mobile learning environment. Based on the original network $R H$, the network was simplified; assuming $o_{i j}$ represents the weight of edge $r_{i j}$, then the keyword co-occurrence adjacency matrix $O G$ of the network can be expressed as Formula 13:

$$
O G=\left[\begin{array}{cccc}
o_{11} & o_{12} & \cdots & o_{m 1} \\
o_{21} & o_{22} & \cdots & o_{m 2} \\
\vdots & \vdots & \ddots & \vdots \\
o_{m 1} & o_{m 2} & \cdots & o_{m m}
\end{array}\right], 1 \leq i, j \leq m
$$

Assuming: $\omega_{i}$ represents the weight of edge $i, p=\left\{r_{1}, r_{2}, \ldots, r_{l}\right\}$ represents a path, $\left\{\omega_{1}\right.$, $\left.\omega_{2}, \ldots, \omega_{l}\right\}$ represents the weight on $p$, then the weight $\omega(p)$ of information transmission path in the mobile learning environment could be calculated by the Minkowski Distance:

$$
\omega(p)=\left[\sum_{i=1}^{l} \omega_{i}^{s}\right]^{\frac{1}{s}}, s=1, \ldots, \infty
$$

The value range of the parameter $s$ of path weight was determined to be within [1, $\infty$ ). When $s=1$, the distance of $p$ is the sum of the weights of all edges of $p$. When $s=2$, 
the distance of $p$ is calculated using Euclidean distance. When $\mathrm{s} \rightarrow \infty$, the distance of $p$ is the distance of the largest edge of $p$.

When all path weights in the network are lower than parameter $t$, the triangle of $t$ obtained by the Minkowski Distance can satisfy the $1 /$ s triangle inequality principle, then there is:

$$
\omega_{h 1} \leq\left(\frac{n}{\omega_{1}^{s}+\omega_{2}^{s}+\omega_{3}^{s}}\right)^{\frac{1}{s}}, n=1,2,3, \ldots, t
$$

Here, $t$ can be set as any integer within [2, $m-1]$. When $t=2$, the above formula describes the triangle inequality rule; when $t=3$, it describes the quadrilateral inequality. Usually, the value of $s$ takes infinity, and the value of $t$ takes $\mathrm{m}-1$.

The connection relationship of the mobile learning environment was simplified through the weighted matrix and the simplified network, and finally the mobile learning environment of keywords co-occurrence of English fragmentation learning was obtained. Assuming: $P S_{i}$ represents the betweenness centrality of terminal node $i$ of labelled keywords, $h_{f p}$ represents the number of shortest paths from terminal node $f$ to terminal node $p$ of labelled keywords, $m_{f p}^{i}$ represents the number of shortest paths that pass through terminal node $i$ in the $h_{f p}$ shortest paths from $f$ to $p$, then, calculation formula of the betweenness centrality of terminal nodes is given by Formula 16:

$$
P S_{i}=\sum_{f \neq i \neq p} \frac{m_{f p}^{i}}{h_{f p}}
$$

Keyword with higher $P S_{i}$ indicates that the corresponding terminal node is more important in the mobile learning environment, meaning that the study content of English fragmentation learning is of higher research value, and learners can carry out learning according to the resource recommendation results of the keywords.

\section{Simulation and experimental results}

In the experiment conducted in this research, the size of the mobile learning network is 3000 . Figure 2 shows the dissemination of learning information in the mobile learning network when the average degree is 5, 7, and 9, respectively. The horizontal axis is the time moment, and the vertical axis is the corresponding cumulative number of mobile terminal nodes. According to the figure, the cumulative number of mobile terminal nodes decreases with the increase of average degree. After reaching a certain time length, the number of terminal nodes receiving English fragmentation learning information and performing mobile learning reaches the maximum and will not decrease, indicating that the dissemination of English fragmentation learning information is different from the spread of viruses in the traditional sense, that is, the terminal nodes have information immunity, but learners are also willing to receive part or all of the learning information after the learning is completed so that they can consolidate learning and reinforce the knowledge. 


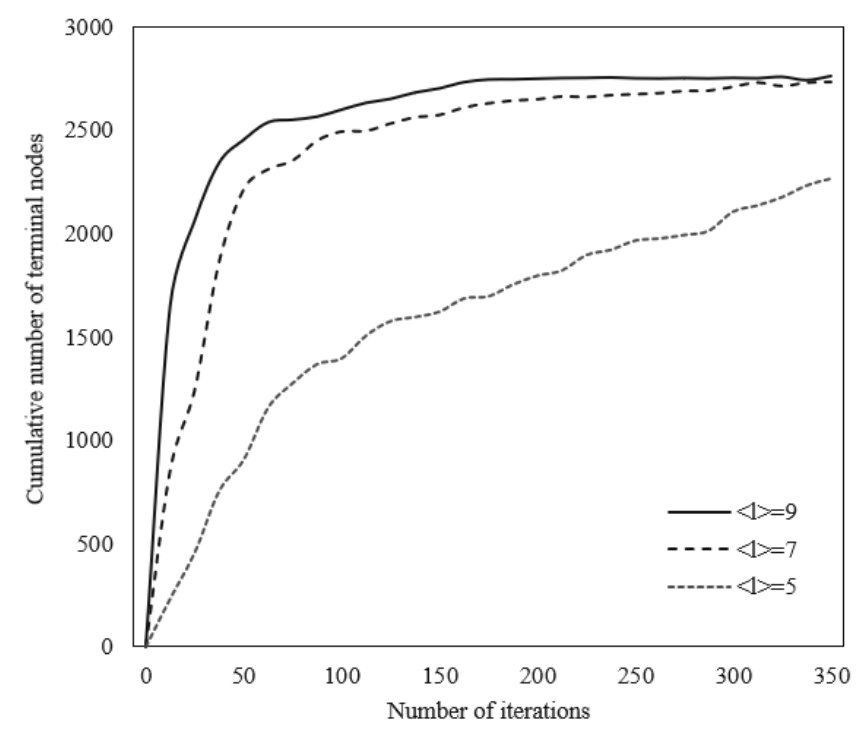

Fig. 2. The dissemination of learning information in the mobile learning network

Table 1 shows the dissemination of learning information in the mobile learning network in different time periods. In the constructed mobile learning network, it only needs $3.4 \%$ of the important terminal nodes to disseminate learning information to meet the requirements.

Table 1. The dissemination of learning information in the mobile learning network in different time periods

\begin{tabular}{|l|c|c|c|}
\hline \multicolumn{1}{c|}{$\begin{array}{c}\text { Experiment condition } \\
\text { Serial number }\end{array}$} & $\mathbf{1}$ & $\mathbf{2}$ & $\mathbf{3}$ \\
\hline$t 1$ & 0 & 1 & 0 \\
\hline$t 2$ & 2335 & 4528 & 4627 \\
\hline$t 3$ & 3248 & 5218 & 6015 \\
\hline$t 4$ & 3758 & 5284 & 6928 \\
\hline$t 5$ & 4021 & 6235 & 7349 \\
\hline$t 6$ & 4235 & 6297 & 7759 \\
\hline$t 7$ & 4516 & 6682 & 8019 \\
\hline$t 8$ & 4825 & 6829 & 8217 \\
\hline$t 9$ & 5017 & 7008 & 8537 \\
\hline$t 10$ & 5186 & 7352 & 8629 \\
\hline$t 11$ & 5316 & 7492 & 8849 \\
\hline$t 12$ & 5476 & 7431 & 8619 \\
\hline$t 13$ & 5819 & 7614 & 9172 \\
\hline$t 14$ & 5816 & 7649 & 9027 \\
\hline$t 15$ & 5966 & 7754 & 9267 \\
\hline
\end{tabular}


Table 2 gives the co-occurrence adjacency matrix of high-frequency keywords in mobile learning. The matrix is a symmetric matrix, values on the diagonal line of the matrix are all 0 , indicating that all keywords have no correlation with themselves. The values of keywords "English listening" and "English speaking" are 1, indicating that there is an edge between them. The values of keywords "English listening" and "English reading" are 0 , indicating that there is no edge between them. Table 3 shows the co-occurrence weighted matrix of high-frequency keywords in mobile learning.

Table 2. Co-occurrence adjacency matrix of high-frequency keywords in mobile learning

\begin{tabular}{|l|c|c|c|c|c|c|}
\hline \multicolumn{1}{|c|}{ Keyword } & Listening & Speaking & Vocabulary & Writing & Reading & Grade test \\
\hline Listening & 0 & 1 & 0 & 1 & 0 & 1 \\
\hline Speaking & 0 & 0 & 1 & 1 & 1 & 1 \\
\hline Vocabulary & 0 & 1 & 0 & 1 & 0 & 0 \\
\hline Writing & 0 & 0 & 1 & 0 & 1 & 1 \\
\hline Reading & 1 & 1 & 0 & 1 & 0 & 1 \\
\hline Grade test & 1 & 0 & 1 & 0 & 0 & 0 \\
\hline
\end{tabular}

Table 3. Co-occurrence weighted matrix of high-frequency keywords in mobile learning

\begin{tabular}{|l|c|c|c|c|c|c|}
\hline \multicolumn{1}{|c|}{ Keyword } & Listening & Speaking & Vocabulary & Writing & Reading & Grade test \\
\hline Listening & 0.264 & 0.06 & 0.087 & 0.057 & 0 & 0.624 \\
\hline Speaking & 0 & 0.274 & 0.285 & 0 & 0.172 & 0.318 \\
\hline Vocabulary & 0.428 & 0.192 & 0 & 0.435 & 0.395 & 0 \\
\hline Writing & 0.751 & 0.625 & 0.492 & 0.513 & 0 & 0.428 \\
\hline Reading & 0.372 & 0.295 & 0.482 & 0 & 0.457 & 0 \\
\hline Grade test & 0 & 0.572 & 0.296 & 0.482 & 0.735 & 0.618 \\
\hline
\end{tabular}

In order to verify the accuracy of the keyword frequency count algorithm used in the experiment, from the dataset containing 10247 keywords of English fragmentation learning, 16 keywords were extracted randomly, including: listening, speaking, vocabulary, writing, reading, grade test, grammar, business English, English teacher qualification certificate, bilingual news, classical literature appreciation, professional English, CET-4 \& CET-6, slow-speed English, listening and speaking training, and TOEFL and IELTS. To ensure the accuracy of statistics, multiple experiments were conducted; the manual labelling results and execution results of the proposed algorithm were compared, as shown in Table 4, according to the data in the table, the experimental results of the two methods were basically consistent, which has verified the effectiveness of the proposed algorithm.

In order to verify the accuracy of the keyword frequency count algorithm used in the experiment, 20 groups of keyword pairs were randomly selected from the high-frequency keyword set containing 208 words. To ensure the accuracy of manual statistics, manual count was repeatedly performed in the experiment, the labels were marked and recorded, and the results were compared with the algorithm execution results. Due to the limited space of the paper, only 10 groups of keyword count results are listed here, and the comparison effect is shown in Table 4. 
Table 4. Statistics of the frequency of randomly extracted keywords

\begin{tabular}{|c|c|c|}
\hline Keyword $\quad$ Frequency & Experimental results & Manual labelling \\
\hline Listening & 125 & 128 \\
\hline Speaking & 75 & 74 \\
\hline Vocabulary & 66 & 68 \\
\hline Writing & 25 & 58 \\
\hline Reading & 24 & 45 \\
\hline Grade test & 22 & 32 \\
\hline Grammar & 21 & 26 \\
\hline Business English & 19 & 24 \\
\hline English teacher qualification certificate & 18 & 20 \\
\hline Bilingual news & 16 & 18 \\
\hline Classical literature appreciation & 14 & 16 \\
\hline Professional English & 14 & 14 \\
\hline CET-4 \& CET-6 & 12 & 12 \\
\hline Slow-speed English & 11 & 10 \\
\hline Listening and speaking training & 9 & 8 \\
\hline TOEFL and IELTS & 6 & 4 \\
\hline
\end{tabular}

According to Table 5, the manual labels are consistent with the execution results of the proposed algorithm, which can prove that the statistical results of the proposed algorithm are accurate, that is, the constructed high-frequency keyword co-occurrence matrix is accurate and effective.

Figure 3 shows the construction results of the mobile learning environment for English fragmentation learning. In the graph, the higher the frequency of a keyword, the more the terminal nodes connected to it, and the larger the area of the circle. From the graph, we can intuitively distinguish the high-frequency keywords of mobile learning, the hot words of learners, and the relationships between them. 


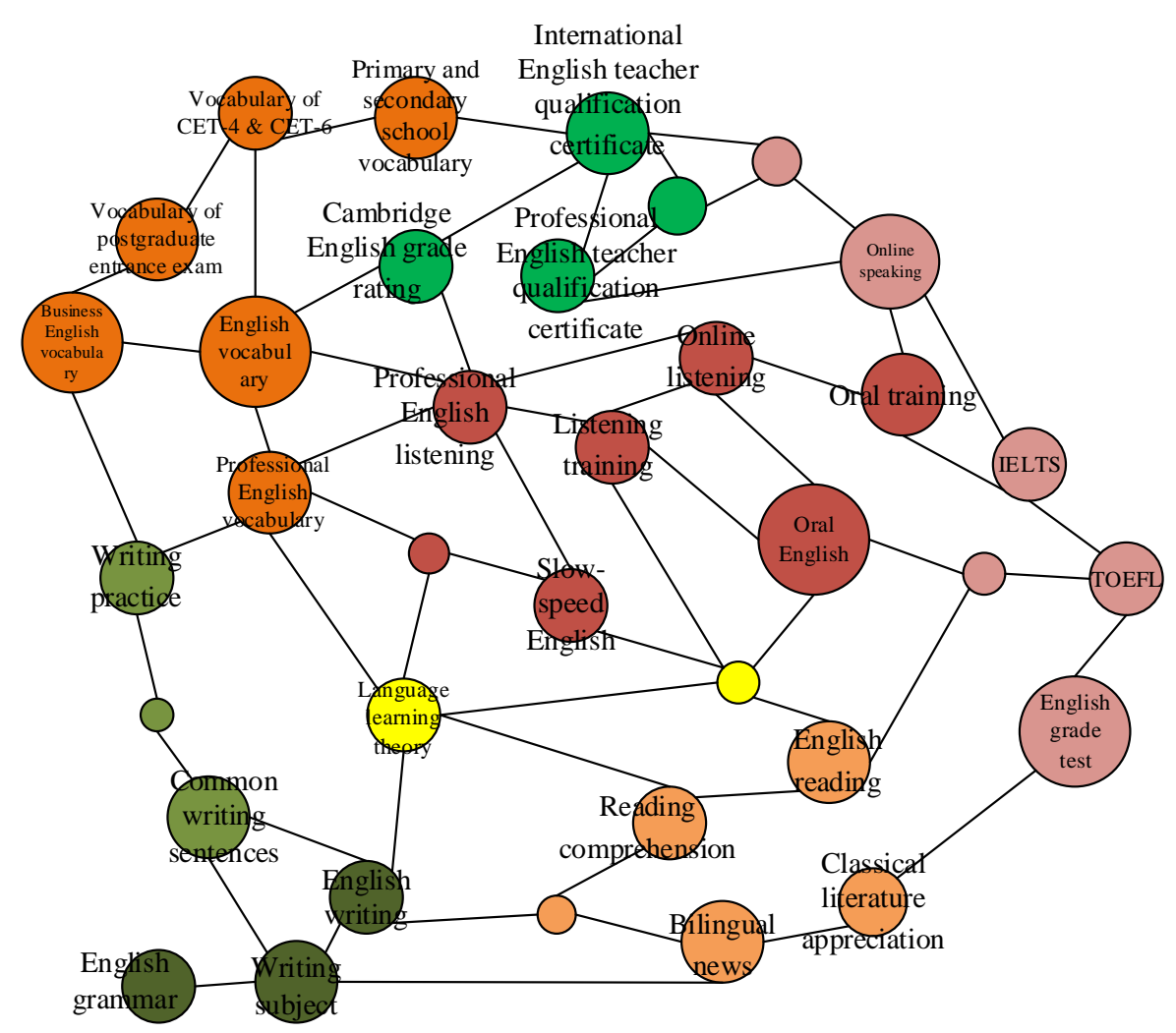

Fig. 3. Construction of mobile learning environment for English fragmentation learning

Table 5. Statistics of co-occurrence frequency of randomly extracted keyword pairs

\begin{tabular}{|c|c|c|}
\hline $\begin{array}{ll}\text { Keyword } & \text { Frequency } \\
\end{array}$ & Experimental results & Manual labelling \\
\hline (Listening, Slow-speed English) & 7 & 8 \\
\hline (Reading, Bilingual news) & 9 & 11 \\
\hline (Grade test, CET-4 \& CET-6) & 1 & 1 \\
\hline (English for going abroad, TOEFL IELTS) & 3 & 5 \\
\hline (Vocabulary, Business English) & 12 & 15 \\
\hline (Reading comprehension, Classical literature appreciation) & 6 & 7 \\
\hline (Professional English, teacher qualification certification) & 3 & 5 \\
\hline (Original-sound English Listening, American Education) & 4 & 2 \\
\hline (Mobile learning, Online listening training) & 6 & 4 \\
\hline (Online teaching, English writing skills) & 2 & 1 \\
\hline
\end{tabular}




\section{Conclusion}

This paper researched the construction of mobile learning environment based on complex network. At first, it introduced the concept and features of complex network for mobile learning in the context of an English fragmentation learning environment, constructed a learning information dissemination model, and proposed an immune strategy under the condition that terminal nodes won't perform similar repeated learning after completing mobile learning. Then, the paper built the keyword co-occurrence adjacency matrix and weighted matrix, and elaborated on the construction process of a mobile learning environment based on keyword co-occurrence. After that, the experimental results showed the dissemination status of learning information in the mobile learning network during different time periods, and gave the constructed co-occurrence adjacency matrix and weighted matrix of high-frequency keywords of mobile learning. By comparing the experimental results of the frequency of randomly extracted keywords and the co-occurrence frequency of randomly extracted keyword pairs, it's verified that the constructed high-frequency keyword co-occurrence matrix is accurate and effective. Finally, construction results of the mobile learning environment of English fragmentation learning were given, and the proposed strategy of mobile learning environment construction was proved to be accurate and usable.

\section{Acknowledgment}

Supported by the Fundamental Research Funds for the Central Universities "POABased Design and Practice of ESP Teaching "(2020MS142).

\section{$7 \quad$ References}

[1] Purnomo, A., Kurniawan, B., Adi, K.R. (2020). Expanding Learning Environment through Mobile Learning, International Journal of Emerging Technologies in Learning, 15(7): 123131. https://doi.org/10.3991/ijet.v15i07.13215

[2] Díaz, J.E.M. (2020). Virtual World as a Complement to Hybrid and Mobile Learning, International Journal of Emerging Technologies in Learning, 15(22): 267-274. https://doi.org/10. 3991/ijet.v15i22.14393

[3] Radhakrishnan, M., Akila, A. (2021). Personalized mobile learning and course recommendation system. International Journal of Mobile and Blended Learning (IJMBL), 13(1): 38-48. https://doi.org/10.4018/IJMBL.2021010103

[4] Zhang, Y., Zhang, X. (2021). Price Learning-based Incentive Mechanism for Mobile Crowd Sensing. ACM Transactions on Sensor Networks (TOSN), 17(2): 1-24. https://doi.org/10.11 45/3447622

[5] Todoranova, L., Penchev, B. (2021). Perspectives for mobile learning in higher education in Bulgaria. Digital Transformation, Cyber Security and Resilience of Modern Societies, 84: 441. https://doi.org/10.1007/978-3-030-65722-2_28

[6] Moldovan, A.N., Muntean, C.H. (2020). DQAMLearn: Device and QoE-Aware adaptive multimedia mobile learning framework. IEEE Transactions on Broadcasting, 67(1): 185200. https://doi.org/10.1007/978-3-030-65722-2_28 
[7] Koomson, W.K. (2018). Mobile learning: Application of Whatsapp messenger as a learning tool in a university distance learning program in Ghana. International Association for Development of the Information Society.

[8] Hamzah, A., Persada, A.G., Hidayatullah, A.F. (2018). Towards a framework of mobile learning user interface design. In Proceedings of the 2018 2nd International Conference on Education and E-Learning, 1-5. https://doi.org/10.1145/3291078.3291080

[9] Jia, J., Zhang, B. (2018). Design guidelines for mobile MOOC learning — an empirical study. in international conference on blended learning. Blended Learning. Enhancing Learning Success-11th International Conference, ICBL, 10949: 347-356. https://doi.org/10.1007/978 -3-319-94505-7_28

[10] Park, E., Han, J., Kim, K.J., Cho, Y., del Pobil, A.P. (2018). Effects of screen size in mobile learning over time. In Proceedings of the 12th International Conference on Ubiquitous Information Management and Communication, 1-5. https://doi.org/10.1145/3164541.31646 $\underline{25}$

[11] Kajonmanee, T., Chaipidech, P., Srisawasdi, N., Chaipah, K. (2020). A personalised mobile learning system for promoting STEM discipline teachers' TPACK development. International Journal of Mobile Learning and Organisation, 14(2): 215-235. https://doi.org/1 $\underline{0.1504 / \mathrm{IJMLO} .2020 .10026336}$

[12] Moedjiono, S., Kusdaryono, A. (2018). Application development based on mobile learning framework on rice and vegetable agriculture. In 2018 Third International Conference on Informatics and Computing (ICIC), 1-6. https://doi.org/10.1109/IAC.2018.8780414

[13] Cai, W., Chen, Q. (2018). An experimental research of augmented reality technology from the perspective of mobile learning. In 2018 IEEE International Conference on Teaching, Assessment, and Learning for Engineering (TALE), 912-915. https://doi.org/10.1109/TALE .2018 .8615146

[14] Jingjing, W.U. (2021). Research on the key technologies of mobile platform development of network ideological and political education innovation based on e-class. In 2021 6th International Conference on Smart Grid and Electrical Automation (ICSGEA), 431-434. https://doi.org/10.1109/ICSGEA53208.2021.00104

[15] Li, X.T. (2017). Application of network media mobile platform in college political education based multi dimension analysis. Boletin Tecnico/Technical Bulletin, 55(6): 179-186.

[16] Zhang, D.G., Liu, S., Zhang, T., Liang, Z. (2017). Novel unequal clustering routing protocol considering energy balancing based on network partition \& distance for mobile education. Journal of Network and Computer Applications, 88: 1-9. https://doi.org/10.1016/j.jnca.2017 .03 .025

[17] Chen, S.Y., Huang, Y.M. (2013). Establishment and application for a mobile learning communities system over cloud network: A case study of digital archives resource into outdoor environmental education. Journal of Internet Technology, 14(6), 985-996.

[18] Chen, L., Zhu, W. (2021). Autonomous mobile learning model of cloud education based on intelligent algorithm of wireless network communication. Wireless Communications and Mobile Computing, 2021. https://doi.org/10.1155/2021/1144767

[19] Ba, Y., Qi, L. (2021). Construction of WeChat mobile teaching platform in the reform of physical education teaching strategy based on deep neural network. Mobile Information Systems, 2021. https://doi.org/10.1155/2021/3532963

[20] Li, M. (2019). Construction of mobile network education data analysis platform based on distributed architecture. In 2019 International Conference on Virtual Reality and Intelligent Systems (ICVRIS), 432-436. https://doi.org/10.1109/ICVRIS.2019.00111 
[21] Mjølsnes, S.F., Olimid, R.F. (2017). Introducing mobile network security experiments to communication technology education. In IFIP World Conference on Information Security Education, 74-83. https://doi.org/10.1007/978-3-319-58553-6_7

[22] Simonova, I., Poulova, P. (2015). Social networks and mobile devices in higher education: pilot project. In 2015 IEEE 39th Annual Computer Software and Applications Conference, 2: 851-856. https://doi.org/10.1109/COMPSAC.2015.192

[23] Amaratunga, T., Ranasinghe, D.N. (2020). Educational content delivery utilizing opportunistic networking over mobile devices. In 2020 20th International Conference on Advances in ICT for Emerging Regions (ICTer), 47-52. https://doi.org/10.1109/ICTer51097 .2020 .9325432

[24] Chen, L., Zhu, W. (2021). Autonomous mobile learning model of cloud education based on intelligent algorithm of wireless network communication. Wireless Communications and Mobile Computing, 2021. https://doi.org/10.1155/2021/1144767

[25] Zhen, L. (2021). An English Mobile Learning Platform Embedded in GSM-R Wireless Network Communication. Security and Communication Networks, 2021. https://doi.org/10. $1155 / 2021 / 8228300$

[26] Zhang, C., Zhong, J. (2018). Construction of mobile learning model based on network collaborative inquiry from Bigdata. In MATEC Web of Conferences, 232: 01032. https://doi .org/10.1051/matecconf/201823201032

\section{Author}

Jie Zhao, female, lecturer, was born on November 18, 1980 in Baoding City, Hebei Province, China. She received her B.A degree in English from Hebei University of Technology in 2002 and M.A degree majoring in English Language and Literature from Hebei University in 2005. She has been teaching English for 16 years, including College English, English Grammar, The Latest Survey of the United Kingdom and the United States. She has published several papers on second language acquisition.

Article submitted 2021-12-18. Resubmitted 2022-01-17. Final acceptance 2022-01-19. Final version published as submitted by the author. 